\title{
VARIAÇÃO TEMPORAL DO INTERVALO HÍDRICO ÓTIMO DE UM LATOSSOLO VERMELHO DISTROFÉRRICO SOB SISTEMAS DE PLANTIO DIRETO ${ }^{(1)}$
}

\author{
Cássio Antonio Tormena ${ }^{(2)}$, Marcelo Alessandro Araújo ${ }^{(3)}$, Jonez \\ Fidalski $^{(4)}$ \& Joaquim Mariano da Costa ${ }^{(5)}$
}

\begin{abstract}
RESUMO
O plantio direto é eficiente no controle das perdas de solo e água, mas o tráfego de máquinas e a ausência de revolvimento podem induzir a compactação superficial dos solos de textura argilosa. O intervalo hídrico ótimo (IHO) traduz os efeitos de sistemas de manejo na melhoria ou na degradação da qualidade física do solo. $\mathrm{O}$ objetivo deste estudo foi quantificar o IHO para discriminar os efeitos de sistemas de manejo em plantio direto na qualidade física do solo. Os tratamentos utilizados foram: plantio direto com sucessão de culturas, plantio direto com rotação de culturas e plantio direto com rotação de culturas e escarificação do solo. Em duas épocas distintas - em outubro de 2002, após a cultura de inverno (trigo), e em abril de 2003, após a cultura de verão (soja) - foram retiradas 44 amostras indeformadas em cada tratamento. Essas amostras foram utilizadas para determinar a curva de retenção de água, a curva de resistência do solo à penetração, a densidade do solo, o IHO e a densidade crítica do solo. A resistência do solo à penetração determinou o limite inferior do IHO em todos os tratamentos e reduziu os seus valores com o aumento da densidade do solo. A densidade crítica do solo não dependeu do sistema de manejo em plantio direto. A variação temporal do IHO nos tratamentos foi dependente da variação da densidade do solo. No plantio direto com rotação, a maior retenção de água em elevados potenciais proporcionou, temporalmente, maiores valores do IHO.
\end{abstract}

Termos de indexação: resistência do solo à penetração, manejo do solo, densidade do solo, rotação de culturas, estrutura do solo.

\footnotetext{
(1) Recebido para publicação em abril de 2006 e aprovado em janeiro de 2007.

(2) Professor do Departamento de Agronomia da Universidade Estadual de Maringá - UEM. Av. Colombo 5790, CEP 87020-900 Maringá (PR). Bolsista do CNPq. E-mail: catormena@uem.br

(3) Engenheiro-Agrônomo, PGA/UEM. E-mail: araujomaa@yahoo.com.br

(4) Pesquisador do Instituto Agronômico do Paraná - IAPAR. Caixa Postal 564, CEP 87701-970 Paranavaí (PR). E-mail: fidalski@iapar.br

(5) Engenheiro-Agrônomo, Fazenda Experimental da COAMO. Especialista em Solos e Meio Ambiente. E-mail: jmariano@coamo.com.br
} 


\title{
SUMMARY: TEMPORAL VARIATION OF THE LEAST LIMITING WATER RANGE OF AN OXISOL UNDER NO-TILLAGE SYSTEMS
}

\begin{abstract}
Despite the efficiency of no-tillage in improving soil and water conservation, the machinery traffic and absence of soil tillage can lead to surface compaction in clayey soils. The Least Limiting Water Range (LLWR) expresses the effects of management systems on the improvement or degradation of soil physical quality. The objective of this study was to quantify $L L W R$ to discriminate the effects of management systems under no-tillage on soil physical quality. The treatments were crop succession under no-tillage, crop rotation under no-tillage and crop rotation under no-tillage plus chiseling. In two different seasons, October 2002, after the winter crop (wheat) and April 2003, after the summer crop (soybean), 44 undisturbed samples were collected in each treatment. The samples were used to determine the soil water retention and soil resistance curve, bulk density, LLWR and critical bulk density. The soil penetration resistance determined the lower limit of LLWR for all treatments and the LLWR declined as the soil bulk density increased. The critical soil bulk density was not influenced by management systems. The temporal variation of $L L W R$ was dependent on soil bulk density variation. The greater soil water retention values at high potentials in the no-tillage with crop rotation treatment led to temporary higher LLWR values.
\end{abstract}

Index terms: soil penetration resistance, soil management, soil bulk density, crop rotation, soil structure.

\section{INTRODUÇÃO}

O sistema plantio direto reduz as perdas de solo, água e nutrientes provocadas pela erosão hídrica, sendo atualmente o principal sistema de manejo de solo utilizado na produção de grãos na região Sul do Brasil. Apesar dessas vantagens, existem limitações relacionadas à compactação superficial do solo, originada da acomodação natural de partículas e do tráfego de máquinas e equipamentos agrícolas. A compactação do solo modifica a distribuição de tamanho de poros (Richard et al., 2001; Klein \& Libardi, 2002), com reflexos na qualidade física do solo para crescimento e produtividade das plantas (Brady \& Weill, 2002).

A rotação de culturas é um dos fundamentos para a qualidade e sustentabilidade da produção em solos sob plantio direto. Do ponto de vista físico, essa técnica de manejo de solo condiciona a melhoria da sua estrutura e possibilita a formação de bioporos com ampla variação de tamanho, que funcionam como rotas alternativas para o crescimento das raízes (Williams \& Weil, 2004). A rotação de culturas constitui uma alternativa para o controle da compactação superficial (Derpsch et al., 1991) e da qualidade física e estrutural do solo em plantio direto. Paralelamente, o controle e a minimização dos efeitos da compactação em plantio direto também podem ser realizados pela escarificação periódica do solo (Câmara \& Klein, 2005), que proporciona condições físico-hídricas mais favoráveis às plantas. No entanto, a longevidade dos efeitos da escarificação pode persistir desde poucos meses (Hamilton-Manns et al., 2002) até alguns anos (Twonlow et al., 1994), com os efeitos de curta duração associados à reconsolidação do solo sob influência do clima e do uso de máquinas (Busscher et al., 2002).
Condições físicas do solo adequadas ao crescimento das plantas resultam de complexas interações entre resistência do solo à penetração (RP), aeração e disponibilidade de água, as quais afetam diretamente o crescimento das plantas. O intervalo hídrico ótimo (IHO) incorpora valores limitantes dessas propriedades físicas do solo às plantas numa única variável, com base no conteúdo de água, levando em conta a variabilidade natural da densidade do solo (Silva et al., 1994; Tormena et al., 1998, 1999). A amplitude do IHO indica a magnitude com que a condição estrutural do solo restringe a qualidade física deste às plantas. Em solos com reduzido IHO, as variações espaciais e temporais de umidade do solo podem predispor as culturas a estresses físicos, por aeração deficiente com solo muito úmido, ou a excessiva RP por ocasião do secamento do solo (Bengough et al., 2006), a exemplo dos estudos de Silva \& Kay (1996) e Lapen et al. (2004).

Nesse cenário, o IHO significa grande avanço em termos de biofísica do solo e é o indicador que melhor traduz a qualidade física do solo às plantas. Várias pesquisas realizadas em diferentes solos, culturas e sistemas de manejo comprovam a pertinência da aplicação do IHO na avaliação da qualidade física do solo (Silva et al., 1994; Tormena et al., 1998, 1999; Imhoff et al., 2001; Wu et al., 2003; Leão et al., 2004).

O IHO pode ser tratado como uma função de pedotransferência, em que a faixa ótima de umidade do solo para o crescimento das plantas depende da densidade do solo (Ds) (Leão et al., 2005). Para cada valor de Ds, um valor de IHO é calculado e definido a partir dos limites superior e inferior de água no solo. Geralmente, aumento de Ds resulta em redução do $\mathrm{IHO}$ em direção a valores de $\mathrm{Ds}$ em que o $\mathrm{IHO}=0$. 
A Ds em que $\mathrm{IHO}=0$ é denominada de densidade crítica do solo (Dsc) (Silva et al., 1994; Imhoff et al., 2001). Quando Ds > Dsc, há indicações de severa degradação estrutural do solo, restringindo o crescimento das plantas. Os valores de Dsc variam com o solo e com as condições de manejo (Silva et al., 1994; Imhoff et al., 2001; Leão et al., 2004; Cavalieri et al., 2006). Potencialmente, sistemas de manejo que propiciam maior freqüência de $\mathrm{Ds}<\mathrm{D}$ sc oferecem menores restrições físicas às plantas.

Na maioria dos agroecossistemas em que o IHO foi estudado, verificou-se que RP é a variável que mais freqüentemente reduz o IHO (Tormena et al., 1998, 1999; Zou et al., 2000; Leão et al., 2005). No entanto, o impacto da RP no IHO depende do valor de RP crítico adotado: por exemplo, valores de $\mathrm{RP}$ entre 2,0 e 3,0 $\mathrm{MPa}$ podem causar redução de cerca de $50 \%$ no crescimento das raízes (Bengough \& Mullins, 1990), e para $\mathrm{RP} \geq 3,0 \mathrm{MPa}$ o crescimento radicular pode ser fortemente impedido (Horn \& Baumgartl, 2000). No entanto, Ehlers et al. (1983) relatam que é possível o crescimento prolífico de raízes com $\mathrm{RP} \geq 3,5 \mathrm{MPa}$, associado à presença de bioporos, que atuam como rotas alternativas para o crescimento das raízes.

Estudos dos impactos de sistemas de manejo na qualidade física e estrutural do solo, em plantio direto, não são freqüentes na literatura. No estádio atual de evolução do sistema de plantio, em que se busca aprimorar a sua qualidade, é necessário conhecer as alterações impostas ao ambiente físico do solo por diferentes estratégias de manejo e em diferentes épocas. Em geral, as avaliações têm sido feitas comparando extremos de sistemas de manejo (plantio direto versus preparo convencional do solo). O objetivo deste estudo foi quantificar o IHO num Latossolo Vermelho distroférrico, cultivado por longo prazo em plantio direto com rotação e sucessão de culturas e com a utilização de escarificação periódica do solo, em duas épocas, após períodos diferenciados de precipitações.

\section{MATERIAL E MÉTODOS}

As amostragens foram realizadas em área experimental localizada no município de Campo Mourão, região noroeste do Paraná, situado a $24^{\circ} 02$ ' 38 " de latitude sul e $52^{\circ} 22$ ' 40 " de longitude oeste, apresentando relevo plano a suave ondulado. Nessa região, o tipo climático dominante, segundo a classificação de Köppen, é o Cfa, que significa subtropical úmido mesotérmico, com médias anuais de temperatura e precipitação de $20^{\circ} \mathrm{C}$ e $1.340 \mathrm{~mm}$, respectivamente. $\mathrm{O}$ solo utilizado neste estudo foi identificado como Latossolo Vermelho distroférrico (Embrapa, 1999). A análise granulométrica na camada de 0-0,20 $\mathrm{m}$ apresentou valores médios de 870,92 e $38 \mathrm{~g} \mathrm{~kg}^{-1}$ de argila, silte e areia, respectivamente.
O experimento foi implantado em 1991 e realizado sob plantio direto, utilizando os seguintes sistemas de manejo como tratamentos: (a) plantio direto com sucessão - sucessão de culturas soja-trigo - PDS; (b) plantio direto com rotação - sistema de rotação milhotrigo-soja-aveia-soja-aveia - PDR; e (c) plantio direto com rotação e escarificação - o sistema de rotação anterior associado à escarificação do solo a cerca de $0,25 \mathrm{~m}$ de profundidade a cada dois anos até o quarto, por meio de um escarificador com cinco hastes espaçadas de 0,25 m e com rolo destorroador (PDE).

A amostragem de solo foi realizada em duas épocas distintas: (a) época 1, em outubro de 2002, após a colheita da cultura de trigo e de um período de menores precipitações; e (b) época 2, em abril de 2003, após a colheita da cultura de soja e de um período de maiores precipitações. Foram selecionados, aleatoriamente, 44 pontos de amostragem por tratamento, em cada época, totalizando 88 amostras por tratamento. Em cada ponto, no centro da camada de $0-0,15 \mathrm{~m}$, foram coletadas amostras com estrutura não-deformada em anéis metálicos $(0,05 \mathrm{~m}$ de diâmetro e $0,05 \mathrm{~m}$ de altura), perfazendo um total de 264 amostras. Em seguida, as amostras foram envoltas em papealumínio, acondicionadas em sacos plásticos e mantidas sob temperatura de aproximadamente $5{ }^{\circ} \mathrm{C}$, até serem processadas no laboratório. Por ocasião das amostragens, os teores de C orgânico do solo em PDS, PDE e PDR foram, respectivamente, de 23, 23 e $24 \mathrm{~g} \mathrm{~kg}^{-1}$ na época 1 e 23,22 e $24 \mathrm{~g} \mathrm{~kg}^{-1}$ na época 2 .

As amostras foram devidamente preparadas e saturadas por meio de elevação gradual de uma lâmina de água em bandejas. Para determinação da curva de retenção, foi adotado o procedimento descrito em Silva et al. (1994). As amostras foram divididas em 11 grupos de 24 amostras, sendo quatro delas por tratamento e época, em cada potencial utilizado. Foram usados os seguintes potenciais: - $0,003,-0,005$, $-0,006,-0,008$ e - $-0,01 \mathrm{MPa}$ numa mesa de tensão e $-0,03,-0,05,-0,07,-0,1,-0,45$ e - $1,5 \mathrm{MPa}$ utilizando pressões aplicadas em câmaras com placas porosas (Klute, 1986). Após atingir o equilíbrio hídrico em cada potencial, as amostras foram pesadas; em seguida, foi feita a determinação da resistência do solo à penetração, utilizando o penetrômetro eletrônico descrito por Tormena et al. (1998). Após determinação da resistência do solo à penetração, as amostras foram secas em estufa a $\pm 105^{\circ} \mathrm{C}$, por $24 \mathrm{~h}$, para determinação da Ds e do teor de água do solo $(\theta)$.

$\mathrm{Na}$ determinação do IHO foram descritas matematicamente a curva de retenção de água (CRA) e a curva de resistência do solo à penetração (CRS). A CRA, expressa pela relação entre o potencial mátrico $(\Psi)$ e o conteúdo de água $(\theta)$, foi descrita matematicamente por meio da função proposta por Ross et al. (1991), conforme a equação 1:

$$
\theta=\mathrm{a} \Psi \mathrm{b}
$$


Para quantificação do IHO, foi incorporada a Ds na descrição matemática da CRA, de maneira que a equação 1 tomasse a forma da equação 2 , similar à utilizada por Silva et al. (1994) e Leão et al. (2005).

$$
\ln (\theta)=\ln (\mathrm{a})+\mathrm{b}^{*} \mathrm{Ds}+\mathrm{c} \ln (\Psi)
$$

em que $\theta$ : conteúdo volumétrico de água $\left(\mathrm{m}^{3} \mathrm{~m}^{-3}\right) ; \Psi$ : potencial matricial (MPa); e $a, b$ e $c$ : coeficientes obtidos no ajuste do modelo. A influência da época nos coeficientes da equação 2 foi descrita como variável qualitativa: época 1 (0) e época 2 (1).

A CRS foi ajustada por meio de um modelo não-linear, conforme Busscher (1990), descrito na equação 3.

$$
\mathrm{RP}=d \theta^{\mathrm{e}} \mathrm{Ds}^{f}
$$

o qual, após transformação logarítmica, resulta na equação 4:

$$
\ln R P=\ln d+e \ln \theta+f \ln \mathrm{Ds}
$$

em que a RP: resistência do solo à penetração $(\mathrm{MPa})$; $\theta$ : umidade do solo $\left(\mathrm{m}^{3} \mathrm{~m}^{-3}\right)$; Ds: densidade do solo $\left(\mathrm{Mg} \mathrm{m}^{-3}\right)$; e $d$, e e $f$ : coeficientes obtidos no ajuste do modelo aos dados. A influência da época nos coeficientes da CRS foi descrita de forma similar àquela utilizada na CRA.

O IHO foi determinado adotando-se os procedimentos descritos em Silva et al. (1994) e Tormena et al. (1998). Os valores críticos de umidade associados com o potencial mátrico, a resistência do solo à penetração $\mathrm{e}$ a porosidade de aeração foram, respectivamente: a capacidade de campo $\left(\theta_{\text {cc }}\right)$ ou conteúdo de água estimado no potencial de -0,01 MPa (Reichardt, 1988); o ponto de murchamento permanente $\left(\theta_{\text {pmp }}\right)$ ou conteúdo de água no potencial de -1,5 MPa (Savage et al., 1996); e o conteúdo de água do solo em que a porosidade de aeração $\left(\theta_{\mathrm{pa}}\right)$ é de $0,10 \mathrm{~m}^{3} \mathrm{~m}^{-3}$ (Grable \& Siemer, 1968). Para a RP foi adotado o valor crítico de $3,5 \mathrm{MPa}$, com base nos resultados de Ehlers et al. (1983). Os valores de $\theta_{\text {cc }}$ e $\theta_{\text {pmp }}$ foram obtidos nos potenciais de - 0,01 e $-1,5 \mathrm{MPa}$, utilizando a CRA; e os valores do teor de água em que a $R P\left(\theta_{\mathrm{rp}}\right)$ atinge o valor crítico de $3,5 \mathrm{MPa}$ foram obtidos por meio da CRS. O valor de $\theta_{\mathrm{pa}}-$ teor de água no solo em que a porosidade de aeração é de $0,10 \mathrm{~m}^{3} \mathrm{~m}^{-3}$ - foi obtido pela expressão [(1-Ds/Dp)-0,1]. Considerou-se o valor médio de densidade de partículas, $\mathrm{Dp}=2,85 \mathrm{Mg} \mathrm{m}^{-3}$, medido nas amostras, conforme Embrapa (1997).

A distribuição dos dados de Ds no modelo de distribuição normal foi avaliada pelo teste de ShapiroWilk, conforme SAS INSTITUTE (1999). A análise de variância dos dados de Ds foi feita utilizando-se o modelo de delineamento inteiramente casualizado, sendo as médias comparadas pelo teste de Tukey $(p<0,05)$. Os ajustes das equações das CRA e CRS foram feitos pelo método de regressão linear com a rotina PROC REG, empregando o programa estatístico SAS (SAS INSTITUTE, 1999).

\section{RESULTADOS E DISCUSSÃO}

A densidade do solo (Ds), o teor de água $(\theta)$ e a resistência do solo à penetração $(\mathrm{RP})$ apresentaram ampla variação de valores (Quadro 1), necessários à parametrização da CRA e da CRS. Os elevados valores dos coeficientes de variação (CV) para RP se devem ao gradiente de $\theta$ e de Ds nas amostras - semelhantes aos verificados em estudos do IHO por Silva et al. (1994), Tormena et al. (1998, 1999), Imhoff et al. (2001) e Leão et al. (2004). O maior CV para a RP na primeira época está associado aos menores valores e à maior variabilidade de $\theta$, condicionando maior amplitude de variação de RP. A Ds, que será utilizada como variável independente nas CRA e CRS e no IHO, apresentou baixos valores de CV e distribuição normal pelo teste de Shapiro-Wilk $(p>0,05)$ em todos os tratamentos e épocas.

Verificou-se que a Ds foi numericamente menor $(p<0,05)$ no tratamento PDE (Quadro 1), indicando o efeito residual da escarificação na redução da Ds, corroborando resultados obtidos por De Maria et al. (1999) e Câmara \& Klein (2005). A variação da Ds com aumento dos valores da época 1 para a época 2 está associada, provavelmente, às diferentes culturas nesses dois períodos e às condições climáticas, que implicaram efeito do tráfego de máquinas, com maior umidade do solo na safra de verão. Essas alterações estruturais do solo, independentemente dos tratamentos, refletiram nos valores médios de RP, que foram menores na RP na época 1 , comparada à época 2 (Quadro 1). A variação temporal da RP foi maior em PDS e PDE, comparando a época 1 à época 2 pelos valores máximos, e em $\mathrm{CV}$, com valores médios de $1,14 \mathrm{Mg} \mathrm{m}^{-3}$ na época 1 e $1,25 \mathrm{Mg} \mathrm{m}^{-3}$ na época 2 . Esses resultados caracterizam a maior resistência mecânica do solo ao desenvolvimento das raízes após o período de menores precipitações (Quadro 1), indicativo de melhor condição estrutural do PDR nos períodos de secamento e molhamento do solo.

Os modelos ajustados explicaram 91, 93 e $91 \%$ da variabilidade de $\theta$ pela CRA e 93,91 e $90 \%$ da variabilidade de RP pela CRS, referente aos tratamentos PDS, PDR e PDE, respectivamente (Quadro 2). A magnitude dos coeficientes dos modelos ajustados à CRA e CRS, considerando unidades similares das variáveis $\theta$, Ds, $\Psi$ e RP, é muito próxima dos valores obtidos por Tormena et al. $(1998,1999)$, considerando serem Latossolos de mesma classe textural. $\mathrm{O}$ teor de água no solo $(\theta)$ foi positivamente influenciado pela Ds e negativamente pelo $\Psi$, igualmente verificados por Tormena et al. (1999) e Betz et al. (1998). A RP variou positivamente com Ds e negativamente $\operatorname{com} \theta$, em acordo com os resultados reportados por Silva et al. (1994), Tormena et al. (1998, 1999), Imhoff et al. (2001) e Leão et al. (2004). O aumento de RP com a Ds pode ser atribuído ao efeito da compactação do solo, o que, segundo Vepraskas (1984), resulta num maior contato ou fricção 
interpartículas. Contrariamente, a redução de RP com $\theta$ deve-se ao efeito lubrificante da água, que reduz a coesão entre as partículas na matriz do solo.
A CRA no tratamento PDR foi dependente da época $(p<0,05)$ e determinou positivamente a retenção de água no solo $(\theta)$ em adição ao efeito da Ds (Quadro 2).

Quadro 1. Momentos estatísticos das variáveis físicas do solo determinadas nas amostras indeformadas, por tratamento e época

\begin{tabular}{|c|c|c|c|c|c|c|}
\hline Variável & Tratamento & Mínimo & Média & Máximo & Desvio-padrão & CV (\%) \\
\hline \multicolumn{7}{|c|}{ Época 1 - outubro de 2002 (após período de menores precipitações) } \\
\hline \multirow[t]{3}{*}{$\mathrm{Ds}\left(\mathrm{Mg} \mathrm{m}^{-3}\right)$} & PDS & 1,04 & $1,14 \mathrm{a}$ & 1,26 & 0,04 & 3,96 \\
\hline & PDR & 1,05 & $1,15 \mathrm{a}$ & 1,26 & 0,05 & 4,52 \\
\hline & PDE & 1,01 & $1,09 \mathrm{~b}$ & 1,27 & 0,05 & 4,76 \\
\hline \multirow[t]{3}{*}{$\theta\left(\mathrm{m}^{3} \mathrm{~m}^{-3}\right)$} & PDS & 0,26 & 0,39 & 0,52 & 0,06 & 16,7 \\
\hline & PDR & 0,29 & 0,39 & 0,49 & 0,06 & 14,24 \\
\hline & PDE & 0,26 & 0,38 & 0,52 & 0,07 & 18,74 \\
\hline \multirow[t]{3}{*}{$\mathrm{RP}(\mathrm{MPa})$} & PDS & 0,57 & 2,78 & 12,75 & 2,39 & 96,83 \\
\hline & PDR & 0,65 & 2,31 & 7,31 & 1,62 & 70,21 \\
\hline & PDE & 0,25 & 1,85 & 6,54 & 1,5 & 81,34 \\
\hline \multicolumn{7}{|c|}{ Época 2 - abril de 2003 (após período de maiores precipitações) } \\
\hline \multirow[t]{3}{*}{$\operatorname{Ds}\left(\mathrm{Mg} \mathrm{m}^{-3}\right)$} & PDS & 1,16 & $1,25 \mathrm{a}$ & 1,33 & 0,04 & 3,46 \\
\hline & PDR & 1,09 & $1,24 \mathrm{ab}$ & 1,34 & 0,06 & 4,66 \\
\hline & PDE & 1,09 & $1,22 \mathrm{~b}$ & 1,32 & 0,05 & 4,14 \\
\hline \multirow[t]{3}{*}{$\theta\left(\mathrm{m}^{3} \mathrm{~m}^{-3}\right)$} & PDS & 0,36 & 0,44 & 0,53 & 0,06 & 12,69 \\
\hline & PDR & 0,33 & 0,43 & 0,51 & 0,05 & 13,21 \\
\hline & PDE & 0,34 & 0,42 & 0,51 & 0,06 & 4,78 \\
\hline \multirow[t]{3}{*}{$\mathrm{RP}(\mathrm{MPa})$} & PDS & 0,98 & 3,36 & 7,22 & 1,43 & 42,45 \\
\hline & PDR & 0,97 & 3,38 & 7,66 & 1,40 & 41,39 \\
\hline & PDE & 1,00 & 2,91 & 5,89 & 1,40 & 48,09 \\
\hline
\end{tabular}

$\mathrm{N}=44$; Ds (densidade do solo); $\theta$ (conteúdo de água); RP (resistência do solo à penetração); CV (coeficiente de variação); PDS (plantio direto com sucessão); PDR (plantio direto com rotação); PDE (plantio direto com rotação e escarificação periódica do solo).

Quadro 2. Equações da curva de resistência do solo à penetração e da curva de retenção de água obtidas a partir dos modelos ajustados aos dados dos três tratamentos

\begin{tabular}{|c|c|c|c|}
\hline Tratamento & Equações & $\mathbf{R}^{2}$ & $\mathbf{F}$ \\
\hline \multicolumn{4}{|c|}{ Curva de retenção de água no solo (CRA) } \\
\hline PDS & $\hat{\theta}=\mathrm{e}^{\left(-2,118+0753^{*} \mathrm{Ds}\right)} \Psi-0,0904$ & 0,91 & 451,80 \\
\hline PDR & $\begin{array}{l}\Lambda \\
\theta=\mathrm{e}^{\left(-2,179+0,816^{*} \mathrm{Ds}+0,798^{*} \text { Época }+0,641^{*} \mathrm{Ds}^{*} \text { Época }\right)} \Psi-0,0822\end{array}$ & 0,93 & 281,85 \\
\hline PDE & $\hat{\theta}=\mathrm{e}^{\left(-2,174+0785^{*} \mathrm{Ds}\right)} \Psi \cdot 0,0940$ & 0,91 & 411,84 \\
\hline \multicolumn{4}{|c|}{ Curva de resistência do solo (CRS) } \\
\hline PDS & 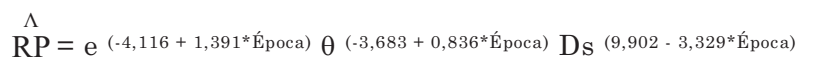 & 0,93 & 221,60 \\
\hline PDR & 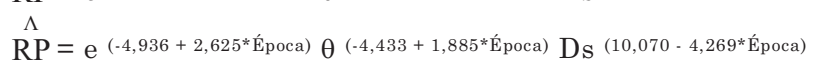 & 0,91 & 158,15 \\
\hline PDE & $\stackrel{\Lambda}{\mathrm{RP}}=\mathrm{e}^{\left(-4,089+0,868^{*} \text { Época }\right)} \theta \theta^{\left(-3,737+0,829^{*} \text { Época }\right)} \mathrm{Ds}^{8,336}$ & 0,90 & 193,19 \\
\hline
\end{tabular}

$\overline{\mathrm{Ds}}$ (densidade do solo, $\mathrm{Mg} \mathrm{m}^{-3}$ ); $\theta$ (conteúdo de água, $\mathrm{m}^{3} \mathrm{~m}^{-3}$ ); RP (resistência do solo à penetração, $\mathrm{MPa}$ ); $\Psi$ (potencial da água no solo, hPa); PDS (plantio direto com sucessão); PDR (plantio direto com rotação); PDE (plantio direto com rotação e escarificação); época é a variável qualitativa com valor igual a zero para a época 1 e valor igual a 1 para a época 2 . 
Em PDE e PDS, a retenção de água no solo ( $\theta$ ) foi positivamente dependente da Ds. As CRS em PDS e PDR mostraram que a época apresentou coeficientes positivos com RP e Ds e negativos com a retenção de água no solo $(\theta)$, caracterizando o aumento da RP da época 1 para a época 2 , associados a aumento da Ds e redução de $\theta$ (Quadro 2). Em PDE, a RP se diferenciou dos demais tratamentos pelo fato de a Ds não ter sido dependente da época, em razão do efeito remanescente da escarificação do solo. Esses resultados indicam que as variações de Ds implicaram maiores valores de RP na época 2 (Quadro 1), em conseqüência da maior compactação do solo, que condiciona maior fricção ou coesão entre as partículas e, ou, agregados na matriz.

Os valores de $\theta_{c c}$ e $\theta_{\text {pmp }}$ nos limites críticos de potenciais de água no solo aumentaram de acordo com a Ds (Figura 1), de forma similar aos resultados de Silva et al. (1994), Tormena et al. (1998, 1999) e Leão et al. (2004), em solos de diferente mineralogia e classe textural. De acordo com Kertzman (1996), em Latossolos argilosos, a compactação ou o aumento da Ds afeta o espaço poroso interagregados, reduzindo o diâmetro dos poros maiores, de modo a se tornarem mais eficientes na retenção de água em elevados potenciais $\left(\theta_{\mathrm{cc}}\right)$. Em baixos potenciais, a relação positiva entre $\theta_{\text {pmp }}$ e Ds deve-se à maior massa de partículas com elevada superfície de adsorção, uma vez que a compactação geralmente não afeta a microporosidade dentro dos agregados. Na figura 1, pode-se verificar que em PDS e PDE não ocorreram alterações expressivas na retenção de água da época 1 para a época 2 , enquanto em PDR houve aumento na retenção de água na época 2 .

Os valores de $\theta_{\mathrm{rp}}$ aumentam com a Ds (Figura 1), justificado pelo fato de que para manter a $\mathrm{RP}=$ 3,5 MPa é necessário aumentar o teor de água do solo $(\theta)$, a fim de compensar a maior friç̧ão entre as partículas resultante do aumento da Ds. Por sua vez, com o aumento da Ds ocorre decréscimo de $\theta_{\mathrm{pa}}$ (Figura 1), indicando a necessidade de maior drenagem do solo para adequada difusão de gases, em virtude da redução dos poros de maior diâmetro. Esses resultados são similares aos obtidos por outros pesquisadores na determinação do IHO (Silva et al., 1994; Tormena et al., 1998, 1999; Imhoff et al., 2001; Leão et al., 2004). Ressalta-se na figura 1 que, independentemente dos tratamentos, $\theta_{\mathrm{rp}}$ foi superior a $\theta_{\mathrm{pmp}}$ em toda a extensão de valores de Ds, a exemplo dos resultados de Imhoff et al. (2001) e Benjamim et al. (2003). Essa constatação corrobora os estudos de Carter et al. (1999), sugerindo que sistemas de manejo com mínimo revolvimento do solo ampliam as possibilidades de limitações às plantas pela RP, de modo que esta assume fundamental importância no controle da disponibilidade de água e da qualidade física do solo.

$O$ valor de $\theta_{\mathrm{pa}}$ manteve-se acima da $\theta_{\mathrm{cc}}$ para $\mathrm{Ds}$ de até $1,24 \mathrm{Mg} \mathrm{m}^{-3}, 1,26 \mathrm{Mg} \mathrm{m}^{-3} \mathrm{e} 1,26 \mathrm{Mg} \mathrm{m}^{-3}$, respectivamente, nos tratamentos $\mathrm{PDS}, \mathrm{PDR}$ e PDE
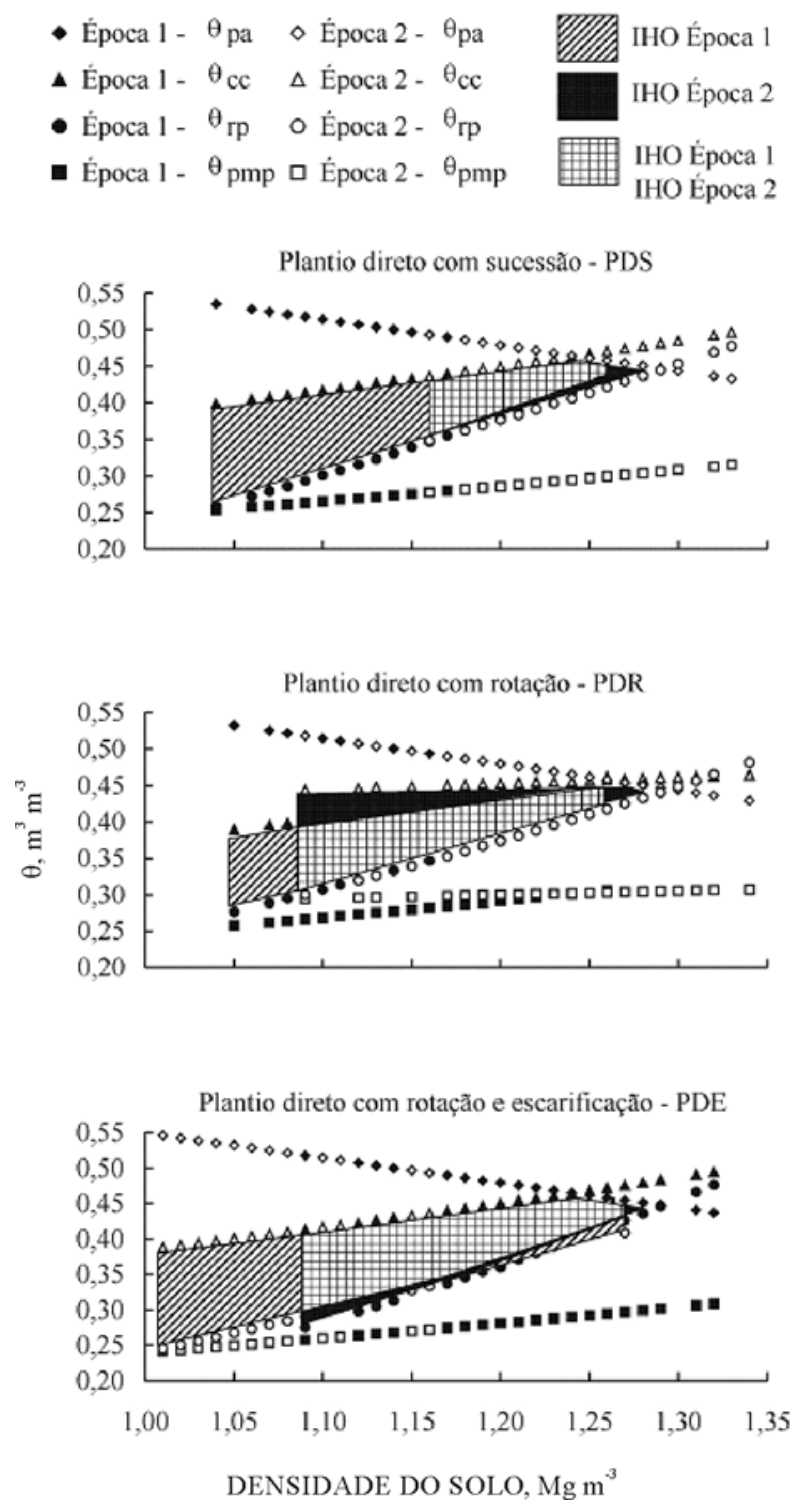

Figura 1. Variação do conteúdo de água $(\theta) \operatorname{com}$ a densidade do solo para os três tratamentos, nos níveis críticos de capacidade de campo $\left(\theta_{\text {cc }}\right)$, ponto de murcha permanente $\left(\theta_{\text {pmp }}\right)$, porosidade de aeração de $0,10 \mathrm{~m}^{3} \mathrm{~m}^{-3}\left(\theta_{\mathrm{pa}}\right)$ e resistência do solo à penetração de $3,5 \mathrm{MPa}\left(\theta_{\mathrm{rp}}\right)$. Áreas hachuradas correspondem ao intervalo hídrico ótimo (IHO) nas épocas de amostragem.

(Figura 1). No tratamento PDS, a redução do valor de Ds em que a aeração $\left(\theta_{\mathrm{pa}}\right)$ substitui a capacidade de campo $\left(\theta_{\text {cc }}\right)$ como limite superior do IHO sugere a redução do volume de macroporos, particularmente de poros de natureza biológica, que são efetivos na drenagem de água do solo em elevados potenciais. Em PDR e PDE, a presença de provável bioporos estáveis e contínuos no solo justifica os maiores valores de Ds em que $\theta_{\mathrm{pa}}<\theta_{\mathrm{cc}}\left(1,27\right.$ a $1,29 \mathrm{Mg} \mathrm{m}^{-3}$; Quadro 1$)$, demonstrando que, apesar da maior compactação, essa 
porosidade se mantém efetiva no processo de drenagem de água e difusão de gases no solo. A maior ocorrência de valores de Ds em que $\theta_{\mathrm{pa}}<\theta_{\mathrm{cc}}$ sugere que, em PDS, há maior risco de as culturas sofrerem estresses por aeração em condições de elevada umidade do solo. Nesses solos, condições anóxicas têm sido indicadas como provável causa de doenças na fase de crescimento inicial das culturas, a exemplo dos resultados reportados por Allmaras et al. (2002).

A variação do IHO para os tratamentos PDS, PDR e PDE nas épocas 1 e 2 manteve relação negativa com a Ds (Figura 2), a exemplo do observado em Silva et al. (1994), Tormena et al. (1999), Leão et al. (2005) e Cavalieri et al. (2006). Os valores do IHO não se diferenciaram entre os tratamentos a partir de $\mathrm{Ds}_{\mathrm{s}}=$ $1,26 \mathrm{Mg} \mathrm{m}^{-3}$, correspondendo aos valores máximos observados na época 1 (Quadro 1). Esses resultados revelam que os valores máximos de Ds na época 2 dos tratamentos não influenciaram o comportamento hídrico do solo, sugerindo que, para valores de Ds superiores a esses, o IHO é determinado por propriedades intrínsecas do solo.

$\mathrm{Na}$ época 1 , os valores de IHO foram superiores a $0,02 \mathrm{~m}^{3} \mathrm{~m}^{-3}$ (Figura 2). O tratamento PDE apresentou valores do IHO superiores aos do PDR; e o PDS, valores extremos do IHO nas Ds de 1,04 e 1,24 $\mathrm{Mg} \mathrm{m}^{-3}$, respectivamente, em relação aos tratamentos $\mathrm{PDE}$ e PDR, caracterizando a maior redução do IHO em PDS com o aumento da Ds. Na amostragem realizada na época 2, os maiores valores de IHO ocorreram em PDE e PDR, comparados ao PDS até Ds $=1,25 \mathrm{Mg} \mathrm{m}^{-3}$, a partir da qual os valores de IHO foram semelhantes, independentemente dos tratamentos.

Variações significativas do IHO em PDS foram descritas matematicamente pela variação da Ds

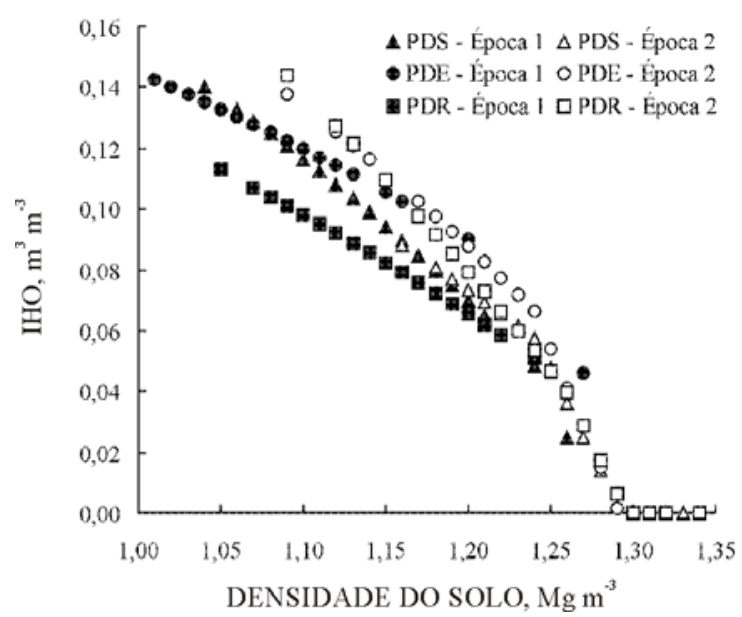

Figura 2. Variação do intervalo hídrico ótimo (IHO) com a densidade do solo para os três tratamentos e duas épocas. PDS (plantio direto com sucessão), PDR (plantio direto com rotação) e PDE (plantio direto com rotação e escarificação).
(Figuras 1 e 2; Quadros 1 e 2). Em PDR, as variações do IHO ocorreram associadas à retenção de água para valores de Ds em que $\theta_{\text {cc }}<\theta_{\text {pa }}$, no intervalo de Ds de 1,09 a $1,26 \mathrm{Mg} \mathrm{m}^{-3}$. No PDE, as variações estiveram associadas a RP, com menor influência em Ds entre 1,09 e $1,18 \mathrm{Mg} \mathrm{m}^{-3}$, e contrariamente, para as Ds de 1,18 a $1,27 \mathrm{Mg} \mathrm{m}^{-3}$. Portanto, o comportamento hídrico do solo quantificado pelo IHO mostra que, temporalmente, a retenção de água em $\mathrm{PDS}$ foi dependente da variação da Ds e, em PDR, da variação da qualidade estrutural do solo em elevados potenciais $\left(\theta_{c c}\right)$. Por outro lado, em PDE, o IHO foi determinado basicamente pela RP (Figura 1).

A similaridade do IHO em PDR e PDE na época 2 - a mais restritiva quanto às propriedades físicas e hídricas do solo - indica que a escarificação inicial do solo pouco acrescentou ao efeito biológico proporcionado pela rotação de culturas (Figura 1). Isso comprova quantitativamente a eficiência da técnica de rotação de culturas em relação à escarificação do solo na ampliação da retenção de água $\left(\theta_{\text {cc }}\right)$ (Figura 1$)$, caracterizando o efeito positivo desta técnica na qualidade estrutural do solo. Constatou-se ausência de sinergismo dessas duas técnicas de manejo em plantio direto, rotação de culturas e escarificação do solo associadas, para aumento do IHO das culturas.

A densidade crítica do solo (Dsc), correspondente à Ds em que IHO = 0 foi similar entre PDS, PDR e PDE, com valores aproximados de 1,30 $\mathrm{Mg} \mathrm{m}^{-3}$ (Figura 2), revela que a Dsc não é influenciada pelos tratamentos. Os valores de Dsc são superiores àqueles encontrados por Tormena et al. $(1998,1999)$ em um Latossolo de textura muito argilosa. Essas diferenças estão associadas ao maior valor crítico de RP adotado neste estudo (3,5 MPa), comparado com $\mathrm{RP}=2,0 \mathrm{MPa}$, adotado por Tormena et al. (1998). Apenas na época 2 é que foram constatados valores de Ds $>$ Dsc. A proporção de Ds $>$ Dsc foi de $5 \%$ em PDE e de cerca de $18 \%$ em PDS e PDR, indicando que a escarificação periódica do solo reduz a ocorrência de condições físicas altamente restritivas às plantas, em acordo com Câmara \& Klein (2005). Apesar da semelhança na proporção de Ds > Dsc, é necessário ressaltar que, em PDR e PDE, os valores de $\mathrm{Ds}_{\mathrm{s}}<1,30 \mathrm{Mg} \mathrm{m}^{-3}$ estão distribuídos numa faixa mais ampla de menores valores de Ds (1,09 $\left.\mathrm{Mg} \mathrm{m}^{-3}\right)$ associados a maiores valores do IHO (Figura 1). No entanto, verifica-se em PDS aumento na freqüência de maiores valores de $\operatorname{Ds}\left(>1,15 \mathrm{Mg} \mathrm{m}^{-3}\right)$ e de menores de IHO, comparado a PDR (Figura 1). Esses resultados confirmam a menor estabilidade estrutural do solo em PDS e, temporalmente (época 2), a maior freqüência de menores valores de IHO neste sistema de manejo (Figura 2).

As propriedades físicas do solo, em especial a Ds, apresentam grande variação temporal, principalmente em PDS (Figura 1), indicando que, neste tratamento, a estrutura do solo está mais sujeita aos efeitos do tráfego de máquinas, independentemente dos períodos 
de precipitações que antecederam as épocas 1 e 2 . Em sistemas conservacionistas, como no caso do plantio direto, a tendência é de que Ds se estabilize em longo prazo, situação que está em acordo com Carter et al. (1999); contudo, isso não foi constatado em PDS após 10 anos, podendo ser atribuído ao manejo contínuo de plantas em sucessão de culturas, comparado ao PDR (Quadro 1). A importância do estudo da variação temporal do IHO é útil para evitar interpretações equivocadas, por ter sido realizada em uma época em que a combinação de fatores (climáticos e tráfego após colheita ou plantio) poderia expressar uma condição de qualidade física do solo que não seria aquela mais freqüente durante todo o ciclo das culturas.

A mudança no comportamento hídrico do solo no tratamento PDR em relação à época - especificamente a dependência do efeito de Ds - está relacionada com a condição estrutural do solo, que determina modificações diferenciadas na distribuição e reorganização do sistema poroso, em comparação com os tratamentos PDE e PDS. Além de Ds e da RP, a variável época incorpora fatores relacionados com a distribuição de poros do solo, como os efeitos relativos ao sistema radicular, que intensifica ciclos de secagem e umedecimento, a formação de bioporos e a atividade de mesofauna. Essas observações, associadas aos resultados apresentados nos quadros 1 e 2 , sugerem que em PDR a condição estrutural do solo produziu um sistema poroso cujo tamanho de poros favoreceu a retenção de água na época 2 , refletindo a influência da rotação de culturas na qualidade física e estrutural do solo, ampliando assim a disponibilidade de água às plantas.

\section{CONCLUSÕES}

1. A resistência do solo à penetração determinou o limite inferior do IHO em todos os tratamentos e reduziu os seus valores com o aumento da densidade do solo.

2. A densidade crítica do solo não dependeu do sistema de manejo em plantio direto.

3. A variação temporal do IHO nos tratamentos foi dependente da variação da densidade do solo.

4. No plantio direto com rotação, a maior retenção de água em elevados potenciais aumentou temporalmente o IHO.

\section{LITERATURA CITADA}

ALLMARAS, R.R.; FRITZ, V.A.; PFLEGER, F.L. \& COPELAND, S.M. Impaired internal drainage and Aphanomyces euteiches root rot of pea caused by soil compaction in a fine-textured soil. Soil Till. Res., 1740:1-12, 2002.

BENGHOUGH, A.G. \& MULLINS, C.E. Mechanical impedance to root growth: a review of experimental techniques and root growth responses. J. Soil Sci., 41:341-358, 1990.
BENGOUGH, A.G.; BRANSBY, M.F.; HANS, J.; McKENNA, S.J.; ROBERTS, T. \& VALENTINE, T.A. Root responses to soil physical conditions:Growth dynamics from field to cell. J. Exp. Botany, 57:437-443, 2006.

BENJAMIN, J.G.; NIELSON, D.C. \& VIGIL, M.F. Quantifying effects of soil conditions on plant growth and crop production. Geoderma, 116:137-148, 2003.

BETZ, C.L.; ALLMARAS, R.R.; COPELAND, S.M.\& RANDALL, G.W. Least limiting water range: traffic and long-term tillage influences in a Webster soil. Soil Sci. Soc. Am. J., 62:1384-1393, 1998.

BRADY, N.C. \& WEIL, R.R. The nature and properties of soils. 13.ed. New Jersey, Pearson Education, 2002. 960p.

BUSSCHER, W.J. Adjustment of flat-tipped penetrometer resistance data to common water content. Trans. Am. Soc. Agric. Eng., 3:519-524, 1990.

BUSSCHER, W.J.; BAUER, P.J. \& FREDERICK, J.R. Recompaction of a coastal loamy sand after deep tillage as a function of subsequent cumulative rainfall. Soil Till. Res., 68:49-57, 2002.

CÂMARA, R.K. \& KLEIN, V.A. Propriedades físico-hídricas do solo sob plantio direto escarificado e rendimento da soja. Ci. Rural, 35:813-819, 2005.

CARTER, M.R.; ANGERS, D.A. \& TOPP, G.C. Characterizing equilibrium physical condition near the surface of a fine sandy loam under conservation tillage in a humid climate. Soil Sci., 164:101-110, 1999.

CAVALIERI, K.M.V.; TORMENA, C.A.; VIDIGAL FILHO, P.S.; GONÇALVES, A.C.A. \& COSTA, A.C.S. Efeitos de sistemas de preparo nas propriedades físicas de um Latossolo Vermelho distrófico. R. Bras. Ci. Solo, 30:137$147,2006$.

DE MARIA, I.C.; CASTRO, O.M. \& SOUZA DIAS, H. Atributos físicos do solo e crescimento radicular de soja em Latossolo Roxo sob diferentes métodos de preparo do solo. R. Bras. Ci. Solo, 23:703-709, 1999.

DERPSCH, R.; ROTH, C.H.; SIDIRAS, N. \& KOPKE, U. Controle de erosão no Paraná, Brasil: sistemas de cobertura do solo, plantio direto e preparo conservacionista do solo. Esch Born, GTZ/IAPAR, 1991. 271p.

EHLERS, W.; KÖPKE, U.; HESSE, F. \& BÖHM, W. Penetration resistance and root growth of oats in tilled and untilled loess soil. Soil Till. Res., 3:261-275, 1983.

EMPRESA BRASILEIRA DE PESQUISA AGROPECUÁRIA . EMBRAPA. Manual de métodos de análises de solos. 2. ed. Rio de Janeiro, 1997. 212p.

EMPRESA BRASILEIRA DE PESQUISA AGROPECUÁRIA EMBRAPA. Sistema brasileiro de classificação de solos. Embrapa Produção de Informação; Rio de Janeiro: Embrapa Solos, 1999. 412p.

GRABLE, A.R. \& SIEMER, E.G. Effects of bulk density, aggregate size, and soil water suction on oxygen diffusion, redox potential and elongation of corn roots. Soil Sci. Soc. Am. J., 32:180-186, 1968. 
HAMILTON-MANNS, M.; ROSS, C.W.; HORNE, D.J. \& BAKER, C.J. Subsoil loosening does little to enhance the transition to no-tillage on a structurally degrade soil. Soil Till. Res., 68:109-119, 2002.

HORN, R.\& BAUMGARTL, T. Dynamic properties of soils. In: SUMNER, M.E., ed. Handbook of Soil Science. Boca Raton, CRC Press, 2000. p.A19-A51.

IMHOFF, S.; SILVA, A.P.; DIAS JÚNIOR, M.S. \& TORMENA, C.A. Quantificação de pressões críticas para o crescimento de plantas. R. Bras. Ci. Solo, 25:11-18, 2001.

KERTZMAN, F.F. Modificações na estrutura do solo e no comportamento de um Latossolo Roxo provocadas pela compactação do solo. São Paulo, Universidade de São Paulo, 1996. 176p. (Tese deDoutorado)

KLEIN, V.A. \& LIBARDI, P.L. Densidade e distribuição do diâmetro dos poros de um Latossolo Vermelho sob diferentes sistemas de uso e manejo. R. Bras. Ci. Solo, $26: 857-867,2002$.

KLUTE, A. Water retention: laboratory methods. In: KLUTE, A., ed. Methods of soil analysis - physical and mineralogical methods. Madison, America Society of Agronomy, 1986. p.635-660.

LAPEN, D.R.; TOPP, G.C.; GREGORICH, E.G. \& CURNOE, W.E. Least limiting water range indicators of soil quality and corn production, eastern Ontario, Canada. Soil Till. Res., 78:151-170, 2004.

LEÃO, T.P.; SILVA, A.P.; MACEDO, M.C.M.; IMHOFF, S. \& EUCLIDES, V.P.B. Intervalo hídrico ótimo na avaliação de sistemas de pastejo contínuo e rotacionado. R. Bras. Ci. Solo, 28:415-423, 2004.

LEÃO, T.P.; SILVA, A.P.; PERFECT, E. \& TORMENA, C.A. An algorithm for calculating the least limiting water range of soil using SAS. Agron. J., 97:1210-1215, 2005.

REICHARDT, K. Capacidade de campo. R. Bras. Ci. Solo, 12:211-216, 1988.

RICHARD, G.; COUSIN, I.; SILLON, J.F.; BRUAND, A. \& GUÉRIF, J. Effects of compaction on the porosity of a silt soil: influence on unsaturated hydraulic properties. Eur. J. Soil Sci., 52:49-58, 2001.
ROSS, P.J.; WILLIANS, J. \& BRISTOW, K.L. Equations for extending water-retention curves to dryness. Soil Sci. Soc. Am. J., 55:923-927, 1991.

SAS INSTITUTE. SAS/STAT procedure guide for personal computers. 5.ed. Cary, 1999.

SAVAGE, MJ.; RITCHIE, J.T.; BLAND, W.L. \& DUGAS, W.A. Lower limit of soil water availability. Agron. J., 88:844851, 1996.

SILVA, A.P. \& KAY, B.D. The sensitivity of shoot growth of corn to the least limiting water range of soils. Plant Soil, 184:323-329, 1996.

SILVA, A.P.; KAY, B.D. \& PERFECT, E. Characterization of the least limiting water range. Soil Sci. Soc. Am. J., 58:1775-1781, 1994.

TORMENA, C.A.; SILVA, A.P. \& LIBARDI, P.L. Caracterização do intervalo hídrico ótimo de um Latossolo Roxo sob plantio direto. R. Bras. Ci. Solo, 22:573-581, 1998.

TORMENA, C.A.; SILVA, A.P. \& LIBARDI, P.L. Soil physical quality of a Brazilian Oxisol under two tillage systems using the least limiting water range approach. Soil Till. Res., 52:223-232, 1999.

TWONLOW, S.J.; PARKINSON, R.J. \& REID, I. Temporal changes in soil physical conditions after deep loosening of a silty clay loam in SW England. Soil Till. Res., 31:3147, 1994.

VEPRASKAS, M.J. Cone index of loamy sands as influenced by pore size distribution and effective stress. Soil Sci. Soc. Am. J., 48:1220-1225, 1984.

WILLIAMS, S.M. \& WEIL, R.R. Crop cover root channels may alleviate soil compaction effects on soybean crop. Soil Sci. Soc. Am. J., 68:1403-1409, 2004.

WU, L.; FENG, G.; LETEY, J.; FERGUSON, L.; MITCHELL, J.; McCULLOUGH-SANDEN, B. \& MARKEGARD, G. Soil management effects on the nonlimiting water range. Geoderma, 114:401-414, 2003.

ZOU, C.; SANDS, R.; BUCHAN, G. \& HUDSON, I. Least limiting water range: a potential indicator of physical quality of forest soils. Aust. J. Soil Res., 38:947-958, 2000. 